\title{
Trapped particle makes 3D images
}

A technique in which a small particle is trapped and moved by laser light has been used to produce visual representations of objects in three dimensions, offering key advantages over currently used approaches. SEE LETTER P.486

\section{BARRY G. BLUNDELL}

$\mathrm{D}$ evices known as volumetric displays allow 3D images to be generated in a transparent enclosure. Because these images occupy three dimensions, they exhibit the spatial characteristics that we associate with real-world scenes. The images can be viewed without the need for glasses by many simultaneous observers, and changes in vantage point allow content to be seen from different orientations. On page 486, Smalley et al. ${ }^{1}$ describe an innovative approach to volumetric-display implementation that allows 3D images to be formed in the air, removing the need for a transparent enclosure.

For more than 100 years, volumetric displays have been the subject of extensive research ${ }^{2}$. Although it is relatively easy to make a small (tabletop) display that works fairly well, it is extremely difficult to develop a larger display that works very well. There are two overarching (but often conflicting) problems. The first relates to the techniques that are currently used to produce dynamic images of relatively high visual quality. The second concerns the optical characteristics of the imaging volume, which must allow light emanating from the image to propagate, and emerge from the volume, without distortion - think of the distortion that occurs when light emerges from a tropical-fish tank.

With respect to the first problem, in most volumetric displays, the imaging volume is formed by the cyclic motion of a transparent surface (Fig. 1a). To produce a 3D image, a sequence of image slices is depicted on the surface as it moves through the volume. Given the need to refresh images at least 30 times per second to avoid perceptible flicker ${ }^{3}$, the surface must move rapidly.

The motion of the surface can be either translational (along a straight line) or rotational. When translational motion is used, the dimensions of the imaging volume are limited by mechanical issues arising from the surface's mass and acceleration. In the case of rotational motion, the surface's linear speed increases with distance from the axis of rotation. This impinges on image quality and so can ultimately restrict the diameter of the imaging volume. There is also a 'dead' region
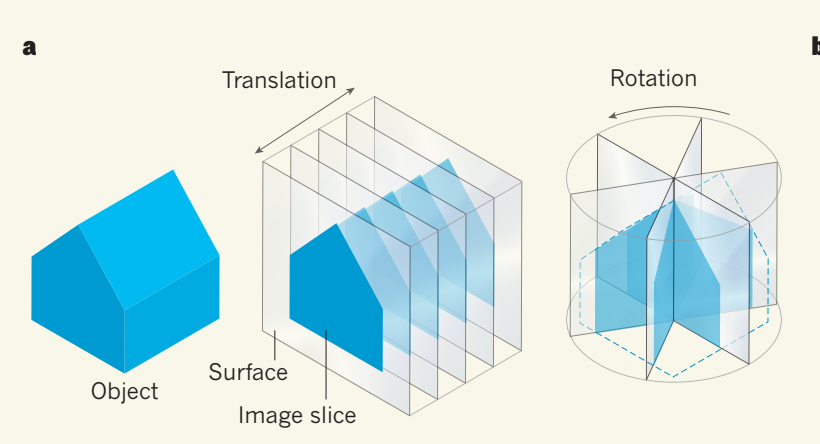

b

Figure 1 | Volumetric-display techniques. a, Devices known as volumetric displays can produce visual representations of objects in three dimensions. They typically use the rapid, cyclic motion of a transparent surface. To generate a 3D image, a sequence of image slices is depicted on the surface as it moves. This motion can be either translational (along a straight line) or rotational. b, Smalley et al. ${ }^{1}$ report an alternative approach in which non-visible laser radiation is used to move a small particle (red arrow). To create an image point, the particle is illuminated with light as it passes through the required position.

in the vicinity of the rotational axis, in which image points cannot be formed ${ }^{4}$.

A further limitation of these displays is that the surface's movement precludes the insertion of haptic probes - tools that recreate the sense of touch by applying forces, motion or vibrations to the user. Such probes can simulate the solidity associated with physical versions of images, so that, for example, virtual clay could be moulded and would feel like real clay.

Smalley et al. sought to overcome all of these difficulties using the photophoretic effect ${ }^{5}$, whereby laser light is used to trap and move small particles (with diameters of 5-100 micrometres). To create a point of light at a given location in 3D space, the authors used nonvisible laser radiation to move a particle, and as the particle passed through the required position, it was illuminated with red, green or blue light (Fig. 1b). The authors suggest that complex, high-fidelity, dynamic images could be formed by introducing parallelism - the simultaneous movement of many particles.

There are at least three key advantages of Smalley and colleagues' approach. First, it does not require the cyclic motion of a surface - movement is restricted to that of low-mass particles. Second, the presence of these particles will have minimal impact on the propagation of light through the imaging volume. And third, because the image is formed in the air, image components can coexist with haptic probes and other interaction tools.

The authors provide several photographs of image content produced using their technique (see Figure 2 of the paper ${ }^{1}$ ). However, these photographs required long exposure times of the order of tens of seconds. For implementing a viable display, there is therefore a pressing need to explore ways of increasing the speed of particle motion and of introducing parallelism such that many image points can be created simultaneously.

The introduction of a high degree of parallelism poses a further challenge, relating to the fact that each point in the imaging volume must be individually accessible. This is reminiscent of an equivalent problem that was encountered in the late 1960s, in connection with a type of 3D display called a photochromicbased volumetric display ${ }^{6,7}$. Another concern is that the insertion of haptic probes into the image volume will probably give rise to shadow regions that will interfere with the propagation of light used for particle motion and illumination. However, the judicious design of such probes would ameliorate this potential problem.

In terms of photorealism, it is unlikely that these devices will ever directly compete with high-end stereoscopic 3D displays. However, despite more than a century of research into volumetric displays, there has been relatively 
little work on exploring ways of capitalizing on key image characteristics. In particular, volumetric displays provide considerable freedom in viewing position, and support both vertical and horizontal motion parallax, which means that observers can move and change their view of an image in a wholly natural way.

Consequently, these devices offer exciting, and largely unexplored, opportunities to advance spatial imaging (in areas such as neurosurgery) and dynamic imaging (in fields including fluid dynamics, robotics and sports training). With regard to the latter, there is a need to better support the visualization of complex forms of 3D motion ${ }^{8}$. Moreover, creating volumetric images in the air enables direct interaction, thereby allowing, for example, 3D design tasks to be carried out in a natural way in $3 \mathrm{D}$ space.

Smalley and colleagues' approach could provide the foundation for the next generation of volumetric displays. Such devices will not only enhance our understanding of complex spatial and geometric dynamics, but also support innovative user interaction.

Barry G. Blundell is with the University of Derby Online Learning (UDOL), University of
Derby, Derby DE22 1GB, UK.

e-mail: barry.blundell@physics.org

1. Smalley, D. E. et al. Nature 553, 486-490 (2018)

2. Luzy, E. \& Dupuis, C. Procédé pour obtenir des projections en relief. French Patent 461,600 (1914).

3. Blundell, B. G. Enhanced Visualization: Making Space for 3-D Images 68 (Wiley, 2007).

4. Blundell, B. G. \& Schwarz, A. Volumetric ThreeDimensional Display Systems 72-91 (Wiley, 2000).

5. Davis, E. J. Aerosol Sci. Technol. 26, 212-254 (1997)

6. Adamson, A. W. Method and apparatus for generating three-dimensional patterns. US Patent 3,609,706 (1971).

7. Adelman, A. H. \& Lewis, J. D. Method and apparatus for generating three-dimensional patterns. US Patent 3,609,707 (1971)

8. Blundell, B. G. 3D Res. 8, 11 (2017).

\section{Ageing-related receptors resolved}

\begin{abstract}
Ageing is a regulated process in which hormones have pivotal roles. Crystal structures of two hormone co-receptors should be informative for drug discovery focused on age-related disorders. SEE ARTICLE P.461 \& LETTER P.501
\end{abstract}

\section{MAKOTO KURO-O}

$\mathrm{I}$ n Greek mythology, three goddesses known as the Fates govern the lifespan of each person. Klotho, Lachesis and Atropos are the spinner, the allotter and the cutter of the thread of life, respectively. So when a genetic mutation was identified in mice that undergo premature ageing ${ }^{1}$, the gene involved was fittingly named klotho. The protein it encodes, $\alpha$-klotho, and a sister protein called $\beta$-klotho, are high-affinity co-receptors for certain members of the fibroblast growth factor (FGF) family of signalling proteins ${ }^{2}$, but their means of action has not been well characterized. Two papers ${ }^{3,4}$ in this issue describe crystal structures of FGF-klotho complexes, not only providing a basis for understanding how klothos act, but also opening up avenues for structure-based drug design.

$a$-Klotho is a membrane-spanning protein expressed predominantly in the kidney, as well as in the brain. Mice lacking $\alpha$-klotho exhibit a range of signs associated with ageing, including hearing loss, impaired cognition and organ atrophy ${ }^{5}$. They also have elevated blood phosphate levels. However, the protein's function on the molecular level was unclear, until mice lacking FGF23 were characterized ${ }^{6}$.

FGF23 is one of the three endocrine FGFs, which act as hormones, secreted by one organ to regulate the function of another. Specifically, FGF23 is secreted from bones after phosphate intake and acts in the kidney to inhibit phosphate reabsorption in urine, thereby maintaining the body's phosphate balance. Mice lacking FGF23 have elevated phosphate levels owing to impaired phosphate excretion, and exhibit features associated with ageing ${ }^{6}$. This striking similarity to mice lacking $\alpha$-klotho led researchers to discover ${ }^{2}$ that $\alpha$-klotho forms a complex with the membrane-spanning protein FGF receptor 1c (FGFR1c), acting as a co-receptor to recruit FGF23 and so triggering FGF signalling

In the first of the current studies, Chen et al. $^{3}$ (page 461) solved the crystal structure of FGF23 in complex with the ligand-binding domain of FGFR1c and the extracellular domain of $\alpha$-klotho. The structure revealed that $\alpha$-klotho (aptly, given its namesake) sends out a long receptor-binding arm (RBA) that acts as a thread to capture the ligand-binding domain of FGFR1c. Indeed, when the authors generated $\alpha$-klotho lacking the RBA, the mutant protein failed to capture FGFR1c or to help FGF23 to activate FGF signalling.

Chen and colleagues showed that FGF23 fits into the groove created between $\alpha$-klotho and FGFR1c. The globular amino-terminal region and the rod-like carboxy-terminal region of FGF23 face FGFR1c and $\alpha$-klotho, respectively (Fig. 1). By promoting formation of this complex, $\alpha$-klotho enables strong interactions between FGF23 and FGFR1c, which otherwise interact only weakly.

Like $\alpha$-klotho, $\beta$-klotho functions as a co-receptor for endocrine FGFs, forming a complex with FGFR1c to bind FGF21, and with FGFR4 to bind FGF19 (refs 7,8). FGF19 is secreted from the intestine after feeding, and acts in the liver to suppress bile-acid synthesis. FGF21 is secreted from the liver following fasting, and acts in fat cells and the brain to induce metabolic adaptation to fasting and responses to stress ${ }^{5}$. Although FGFRs are expressed in a wide range of tissues, the tissuespecific expression of $\beta$-klotho in the liver, fat and brain restricts the target organs of these endocrine FGFs.

In the second study, Lee et al. ${ }^{4}$ (page 501)

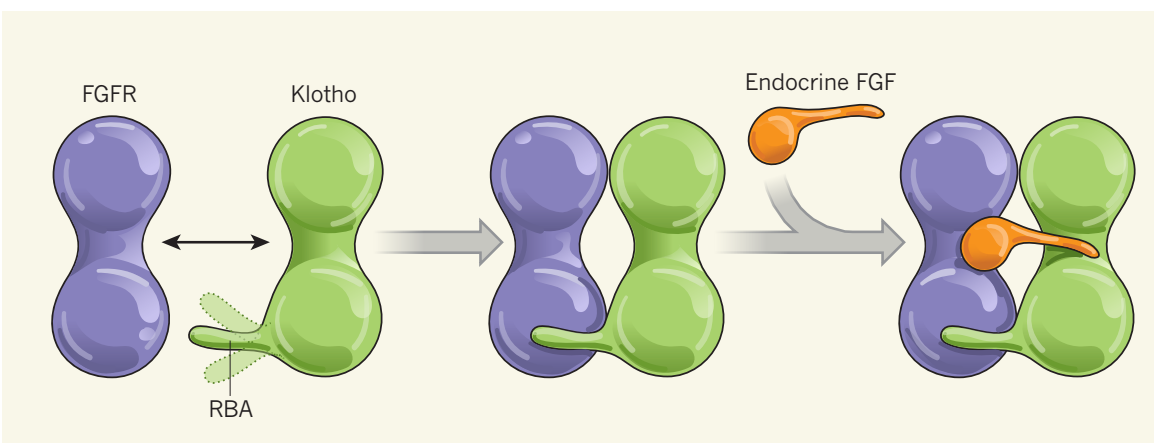

Figure 1 | Structures reveal the mode of action for klotho proteins. Two groups $\mathrm{s}^{3,4}$ have produced crystal structures of the extracellular domains of klotho proteins, either alone, in complex with 'endocrine' fibroblast growth factors (FGFs), or in complex with both endocrine FGFs and the ligandbinding domains of FGF receptors (FGFRs). As this simplified schematic shows, the klotho proteins seem to have an intrinsically disordered receptor-binding arm (RBA) with which they capture FGFRs (interaction indicated by double-headed arrow). The RBA enables formation of a stable complex, with FGFs fitting into the groove between the other two proteins. 